magma. The bathymetry profiles, on the other hand, suffer no such disadvantages - the 7 million year anomaly has a significant effect, the base line (the depth of the oceanic basement away from the ridge) is well known, and the observed profile, being continuous, easily distinguishes local topographic perturbations.

What emerges from the bathymetric comparison is that the model involving a constant spreading rate (the $0.75 \mathrm{~cm}$ $\mathrm{yr}^{-1}$ rate from plate tectonics) wins, at least south of a fracture zone $(75 \mathrm{~km}$ offset) which crosses the survey region. Here, between $30 \mathrm{~km}$ and $150 \mathrm{~km}$ from the central rift, there is insufficient elevation to accommodate a doubling of the spreading rate for 7 million years. For at least the past 40 million years the spreading rate has apparently been less than $1.0 \mathrm{~cm} \mathrm{yr}^{-1}$

North of the fracture zone the situation is quite different, for not even the plate tectonic interpretation applies. To the south, plate tectonic predictions of topography and heat flow agree with observations, but to the north neither do. Within $150 \mathrm{~km}$ of the central rift the topography is too flat. Between 200 and $500 \mathrm{~km}$ to the east, it is too high; but the heat flow there is low, which means that if the high elevation is to be explained by thermal expansion the temperature gradient must increase rapidly within the upper few $\mathrm{km}$.

\section{HORTICULTURE}

\section{Fusarium Patch Disease}

from a Correspondent

AfTER a dry few weeks, lawns in Britain are not looking at their best and, even though autumn rain and applications of fertilizers will restore most turf to a better condition, excessive use of water and fertilizer can easily lead at this time of the year to an outbreak of fusarium patch disease.

This disease, which is caused by Fusarium nivale (Fr.) Ces., is one of the commonest and most damaging diseases of turfgrass in Britain. Outbreaks of the disease can take place in any season, but it is most prevalent in spring and autumn. Humidity, especially at the turf surface, is one of the principal factors at the infection stage of the disease, which is more likely to occur in shaded areas than in warm and overcast conditions. Layers of moist fibre in the surface of the turf encourage infection and usually the first symptoms are yellowish brown patches approximately 1 to 2 inches in diameter. These patches can spread and the disease increases in severity very rapidly if environmental conditions are favourable. In one or two days widespread and severe damage can occur with the possibility of the grass being completely destroyed.

Examination of dying plant parts behind the advancing edge of the mycelium readily reveals large numbers of the sickle-shaped spores which are often formed in sporodochia. These spores are easily carried on the feet, on grass clippings and on machinery and can give rise to fresh outbreaks. The life of the spores seems to be fairly short, and it may be that the original infections take place by development of aggregations of mycelium embedded in grass leaf residues.

The bare areas produced by the disease are very unsightly, and leave the turf open to invasion by dicotyledonous weeds and undesirable grass species. A grass which commonly fills the bare areas is annual meadow-grass (Poa annua $\mathrm{L}$.), which, unfortunately, is particularly susceptible to fusarium patch disease. The presence of this species in many fine turf areas increases the risk of disease and although other species of grass used in turf, including browntop (Agrostis tenuis Sibth) and red fescue (Festuca rubra L.) can be attacked, $P$. annua is often the first to be infected. Some varieties of perennial ryegrass (Lolium perenne L.) are, however, moderately resistant.

In general, increasing nitrogen levels result in an increase in the infection. The disease spreads following the use of fertilizers such as sodium nitrate and nitro-chalk, which tend to make the turf more alkaline. The time at which fertilizer is applied is also important and treatment with any fertilizer that gives readily available nitrogen in September often leads to rapid development of the disease if the weather conditions are favourable. In this case the grass growth is often very lush and the leaves are rather susceptible to mechanical damage.

\title{
Faraday Rotation in Radio Galaxies and Quasars
}

THE origin of the polarization of extragalactic radio sources is tackled in next Monday's Nature Physical Science by R. G. Strom of Leiden Observatory. He has used observations of the linear polarization of a number of sources to examine the relationship between the linear diameter of the sources and the product of the line-of-sight magnetic field and the integrated electron density.

The linear diameter is simply obtained from a consideration of the angular diameter and the redshift, assuming the redshift to be cosmological. The product of the magnetic field and the electron density, on the other hand, is obtained on the assumption that the Faraday effect occurring within the sources is responsible for the observed dependence of linear polarization on wavelength.

Strom has plotted these two quantities for twenty-eight radio galaxies and
Treatment with fungicides can prevent infection and control the disease after it has begun to develop. Fortunately most of the fungicides sold for use on turf in Britain are effective because this disease is one they must control to be commercially viable.

The pattern of disease development described is not the only one possible. Occasionally in Britain, but more frequently in Northern Europe and North America, $F$. nivale is responsible for "pink snow mould", a condition where infection and development take place under snow cover. In this case damage to the turf is revealed when the snow melts, and often mycelium of the fungus can be seen among the dead grass.

\section{Ponetrating fields}

from a Correspondent

IN a new calculation of the capacitance of two metal plates separated by a vacuum, Theophilou and Modinos (Phys. Rev. B, 6, 801; 1972) have managed to avoid making the assumption that charge terminates abruptly at the metal-vacuum interfaces and have shown that the capacitance arising at the interface because of penetrating electric fields is negligible.

As every student of physics knows, the capacitance of a parallel plate capacitor depends on the inverse of the distance between the plates. This honourable result of classical electrostatics remained unassailed until quite recently, when Mead (Phys. Rev. Lett., 6, $545 ; 1961)$ observed that the capacitance of very thin $\mathrm{Ta}-\mathrm{Ta}_{2} \mathrm{O}_{5}-\mathrm{Au}$ sandwiches deviated from the expected value, and that if the plate separation was extrapolated to zero, the capacitance did not

twelve quasars. By assuming that each electron contributing to the electron density comes from an ionized hydrogen atom it is possible to use this plot to obtain for the sources the product of the mass of ionized hydrogen which they contain and the line-of-sight magnetic field. According to Strom this product for about half the sources is $10^{\mathbf{2} \pm 0.5} \mathrm{M}_{\odot}$ gauss. Typical values for the parameters involved in the product may be $10^{7} M_{\odot}$ for the mass of ionized hydrogen and $10^{-5}$ gauss for the line-of-sight field.

An interesting feature of Strom's plot is the general similarity which it shows between the values of the product (mass of ionized hydrogen) $\times$ (line-of-sight magnetic field) for quasars and for radio galaxies. This is of course based on the assumption that the quasar redshifts are cosmological, and Strom says that if, to the contrary, quasars are nearby objects, the good agreement is lost. 